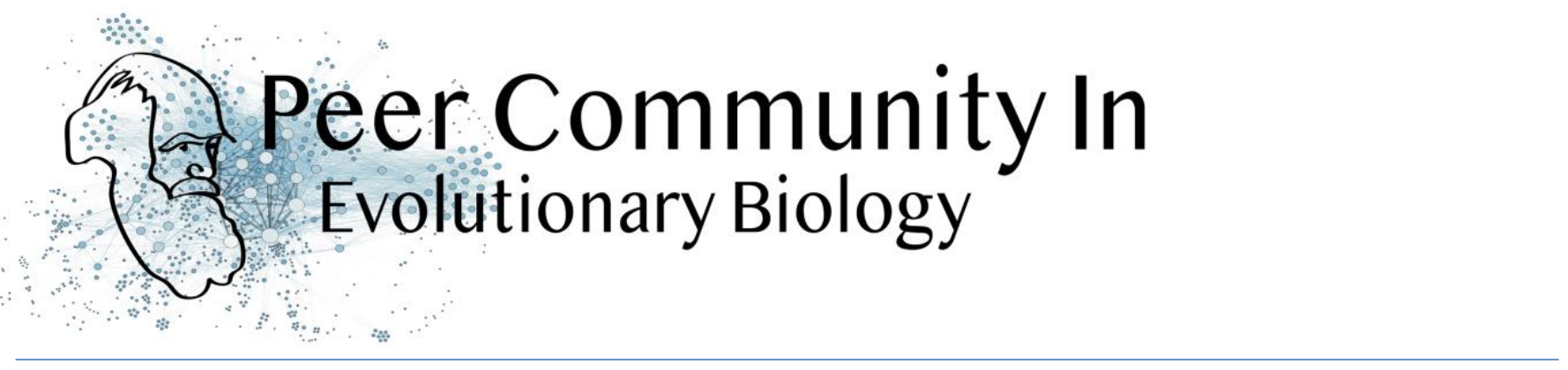

\title{
From populations to
}

subspecies... to species?

Contrasting patterns of local

adaptation in closely-related

\section{taxa and their potential}

Open Access contribution to species

\section{divergence}

Published: 14 May 2019

Copyright: This work is licensed under the Creative Commons

Attribution-NoDerivatives 4.0

International License. To view a

copy of this license, visit

http://creativecommons.org/licen ses/by-nd/4.0/
Emmanuelle Porcher based on reviews by Santiago C. Gonzalez-Martinez, Sophie Karrenberg and 1 anonymous reviewer

A recommendation of:

Sara Marin, Anaïs Gibert, Juliette Archambeau, Vincent

Bonhomme, Mylène Lascoste and Benoit Pujol. Potential adaptive divergence between subspecies and populations of snapdragon plants inferred from QST - FST comparisons (2020), Zenodo, 3628168, ver. 3 peer-reviewed and recommended by Peer Community in Evolutionary Biology. 10.5281/zenodo.3628168

Submitted: 05 August 2018, Recommended: 14 May 2020 Cite this recommendation as:

Emmanuelle Porcher (2020) From populations to subspecies... to species? Contrasting patterns of local adaptation in closely-related taxa and their potential contribution to species divergence. Peer Community in Evolutionary Biology, 100097. 10.24072/pci.evolbiol.100097 


\section{Biol}

Elevation gradients are convenient and widely used natural setups to study local adaptation, particularly in these times of rapid climate change [e.g. 1]. Marin and her collaborators [2] did not follow the mainstream, however. Instead of tackling adaptation to climate change, they used elevation gradients to address another crucial evolutionary question [3]: could adaptation to altitude lead to ecological speciation, i.e. reproductive isolation between populations in spite of gene flow? More specifically, they examined how much local adaptation to environmental variation differed among closely-related, recently diverged subspecies. They studied several populations of two subspecies of snapdragon (Antirrhinum majus), with adjacent geographical distributions. Using common garden experiments and the classical, but still useful, QST-FST comparison, they demonstrate contrasting patterns of local adaptation to altitude between the two subspecies, with several traits under divergent selection in A. majus striatum but none in $A$. majus pseudomajus. These differences in local adaptation may contribute to species divergence, and open many stimulating questions on the underlying mechanisms, such as the identity of environmental drivers or contribution of reproductive isolation involving flower color polymorphism.

\section{References}

[1] Anderson, J. T., and Wadgymar, S. M. (2020). Climate change disrupts local adaptation and favours upslope migration. Ecology letters, 23(1), 181-192. doi: 10.1111/ele.13427 [2] Marin, S., Gibert, A., Archambeau, J., Bonhomme, V., Lascoste, M., and Pujol, B. (2020). Potential adaptive divergence between subspecies and populations of snapdragon plants inferred from QST - FST comparisons. Zenodo, 3628168, ver. 3 peer-reviewed and recommended by Peer Community in Evolutionary Biology. doi: 10.5281/zenodo.3628168 [3] Schluter, D. (2009). Evidence for ecological speciation and its alternative. Science, 323(5915), 737-741. doi: 10.1126/science.1160006

\section{Revision round \#2}




\section{Biol}

Decision on your preprint "Potential adaptive divergence between subspecies and populations of snapdragon plants inferred from Qst-Fst comparisons"

Dear Sarah Marin,

Thank you for submitting a revised version of your manuscript to $\mathrm{PCl}$ Evolutionary Biology, and for answering one critical question so rapidly. This version has been evaluated by two reviewers, whose comments are hopefully attached to this message (if not, let me know). First, I would like to renew my apologies for the extremely long time it took me to return this decision. This is due to the pandemics, but still, this excuse is of no great comfort to you. As you will see, both reviewers found that the new, and significantly modified, version of your article is greatly improved. The question of the calculation of Qst is now solved, and I agree that it does not modify the main results of your study, such that I will be happy to recommend it for $\mathrm{PCl}$ Evolutionary Biology provided you make the few relatively minor changes suggested by both reviewers and myself.

Thank you very much for submitting your preprint for recommendation in $\mathrm{PCl}$ Evolutionary Biology.

Best regards Emmanuelle Porcher

Recommender's comments

Abstract: the words « potential » or « potentially » appear 6 times in the abstract. It is good to be cautious but surely some of these could be reworded (check lines 21-24 out in particular)

Line 249: I am not sure what "linearized" means here

Line 263: replace 'last' with 'latter'

Lines 282-285: this latter analysis seems to be the most comprehensive, and the one you really need to address your main questions. What is the point of the other Mantel tests described above?

Lines 312-317: Could you confirm that the difference in the correlation between trait values and altitude between the two taxa is not due differences in the 


\section{Biol}

variance in altitude across populations for each taxon? Is variance in altitude the same for both taxa?

Lines 332-336: do you mean that the results obtained via the examination of overlapping confidence intervals are fully consistent with those obtained by the bootstrapping method? If so, this is not entirely clear from this sentence.

Lines 384 and 385: replace two instances of "less" with "fewer"

\section{Additional Comments of the managing board:}

Mandatory modifications. As indicated in the 'How does it work?' section and in the code of conduct, please make sure that: -Data are available to readers, either in the text or through an open data repository such as Zenodo (free), Dryad or some other institutional repository. Data must be reusable, thus metadata or accompanying text must carefully describe the data. -Details on quantitative analyses (e.g., data treatment and statistical scripts in R, bioinformatic pipeline scripts, etc.) and details concerning simulations (scripts, codes) are available to readers in the text, as appendices, or through an open data repository, such as Zenodo, Dryad or some other institutional repository. The scripts or codes must be carefully described so that they can be reused. -Details on experimental procedures are available to readers in the text or as appendices. -Authors have no financial conflict of interest relating to the article. The article must contain a "Conflict of interest disclosure" paragraph before the reference section containing this sentence: "The authors of this preprint declare that they have no financial conflict of interest with the content of this article." If appropriate, this disclosure may be completed by a sentence indicating that some of the authors are $\mathrm{PCl}$ recommenders: "XXX is one of the PCI XXX recommenders."

In order to reach a better referencing and greater visibility of your recommended preprint, we suggest you to do the following modifications: - add the following sentence in the acknowledgements: "Version 3 of this preprint has been peerreviewed and recommended by Peer Community In Evolutionary Biology (https://doi.org/10.24072/pci.evolbiol.100097)" 
- in the Zenodo metadata "Notes" you can indicate: "This article has been peerreviewed and recommended by Peer Community In Evolutionary Biology (https://doi.org/10.24072/pci.evolbiol.100097)"

- in the Zenodo metadata "Related article" you can indicate: "Reviewed by PCl Evol Biol 10.24072/pci.evolbiol.100097"

Optional modifications. ==> We advise you to use templates (word docx template and a latex template) to format your preprint in a $\mathrm{PCl}$ style. This is optional. Here is the links of the templates: https://peercommunityin.org/templates/

For word template, please be careful to correctly update all text in these templates (doi, authors' names, address, title, date, recommender first name and family name ...). Please be careful to choose the badges "Open Code" and "Open Data" only if appropriate (in addition to the "Open Access" and "Open PeerReview" badges). If some of the reviewers are anonymous, indicate for example "Albert Ayler and two anonymous reviewer". Indicate in the "cite as" box the right version of your preprint. It is version 3 .

For Latex template, main.tex and sample.bib should be filled. Please be careful to choose the badges "Open Code" and "Open Data" only if appropriate (in addition to the "Open Access" and "Open Peer-Review" badges). Preambule_xxx.tex should be modified (comment lines 115,117 ) to select badges. If some of the reviewers are anonymous, indicate for example "Albert Ayler and two anonymous reviewer". In sample.bib, indicate the right version of your preprint. It is version 3 .

I hope this is clear. Do not hesitate to ask any help if you need. Once you have made these modifications (plus those requested by the recommender), you should upload a new version of the article on the preprint server. Please tell us when you have done so. Thanks.

Preprint DOI: https://doi.org/10.5281/zenodo.3628169

Reviewed by Santiago C. Gonzalez-Martinez, 2020-04-10 10:08 


\section{Biol}

First of all, my apologies for taking so long to do this review. I am happy about how authors addressed my comments and I find that the manuscript is now more comprehensive. Moreover, the new focus considering the two subspecies separately made it more interesting and appealing.

In this second round of revision, I only have a major comment. I am afraid that the equation provided to compute $\mathrm{Qst}, \mathrm{Vb} /(\mathrm{Vb}+2 \mathrm{~h} 2 \mathrm{Vw})$, is wrong. The correct denominator of this equation is the sum of the variance among populations $(\mathrm{Vb})$ and twice the additive variance within-populations, which in your case (full sibs) is twice the family variance $(\mathrm{Vw})$, thus the equation should read: $\mathrm{Vb} /(\mathrm{Vb}+2 \mathrm{Va})$ or $\mathrm{Vb} /(\mathrm{Vb}+4 \mathrm{Vw})$ or $\mathrm{Vb} /(\mathrm{Vb}+2 \mathrm{~h} 2 \mathrm{Vp})$, with $\mathrm{Vp}=\mathrm{Vw}+\mathrm{Vres}$. I tried to check out if this is just an erratum by recomputing Qst from the variance components from Table S3 but Qst values do not seem to fit with those in Figure 4 (Qst values themselves are not reported in the manuscript) in any way I compute them. Please, check this out, as it may imply major revisions to the manuscript.

Minor comments:

Ln 35. Replace "subspecies" by "subspecies divergence".

Ln 49-51. Can you be a bit more specific?

Ln 100-102. Not clear to me why you would not expect shorter internode length at high altitudes, as I would expect than to only height but also growth rate would be lower (but I am not an expert on this, so I am probably wrong). In any case I like very much this paragraph setting the expected trait variation with altitude.

Ln 111. 'based on trait heritability', a bit weird to express it in this way... perhaps better to say 'based on partition of genetic variance'?

Ln 138. I guess that you estimated genetic differentiation using Fst? Can you add this between parentheses?

Ln 159. It is perhaps not very important for this study, as climatic data is only used to characterise the populations, but worldclim does notably bad in mountain regions (in particular for precipitation) and alternative climate databases are preferable. 
Ln 205. I assume that you mean '...the 637 plants that germinated'? (As there should have been $40 \times 24=960$ pots). Please, specify.

Ln 221/230/241. '... plastic container ('block') as a random...'

Ln 231. '...where $V w$ is the family...'

Ln 243. Please, see my major comment above. Hope it is just an erratum!

I think you would need to report Qst values somewhere in the Results (perhaps adding these values to Table S3?). I also miss some discussion on these values and the comparison with values for other plants in the literature.

Ln 323. 'Several traits had similar...'

Ln 366-367. I would say that signatures of potential selection were found for only few traits. Do you think this is because low power of the study (Cls for Qst are large, judging by Figure 4) or does it truly reflect the species' biology?

Ln 389. Should this section not come before the previous one? (As it talks about overall differences in adaptation patterns across subspecies) Overall, the Discussion section is still a bit reiterative and, towards the end, it puts too much emphasis on adaptive divergence. This is surprising as adaptive divergence was found for only a handful of traits in this study. I think that a section devoted to why the study did not identify adaptive divergence in more traits (some of them with obvious adaptive value, e.g. SLA) and why altitude did not seem to be a major factor for adaptive divergence in this system would be desirable.

Figure 2's legend. No need to report the regression fits as they are not significant.

Figure 5 needs updating (these linear correlations were removed from the manuscript, thus should not be reported in this figure). Also, why Fst in the y-axis? All in all, this is a very nice paper and hope that authors will be able to deal with the few revisions that are still needed (and in particular to check out that Qst values are indeed properly computed).

Reviewed by anonymous reviewer, 2020-04-09 13:31 
The manuscript by Marin et al. has changed greatly after the revision, so that many of the previous comments by reviewers do not apply anymore. In my opinion the new version is a clear improvement, based on what I read in the previous reviews. My review below is thus independent from those.

The paper is an interesting contribution to our understanding of plant adaptive variation (or lack thereof) along elevation gradients and of how recently-diverged taxa could be responding differently to similar environmental pressures. The authors use an Fst/Gst approach, based on a common garden and previous field studies, to study patterns of local adaptation of two subspecies of snapdragons. The approach is sensible in cases like this where reciprocal transplants are difficult. The paper is overall clearly written but there are some aspects of the analysis and results that need clarification:

- Values of narrow sense heritabilities are estimated in the greenhouse across all populations within each subspecies, rather than for each population. Please justify why this done, given that all subsequent analyses are based on population comparisons, and that we know that h2 values are populationdependent. The authors mention in line 234 that caution must be taken with h2 values, but it is not clear how they are being cautious in the analyses that follow.

- The text explains that pairwise relationships were tested with Mantel and partial Mantel tests, but legends of Fig 2 and Fig 5 include regression equations and $p$ values - please clarify what the analysis was.

- I would expect that some of the traits included in the analysis are correlated to each other, notably the number of nodes, branches and internode length. The two traits that are found to vary with altitude, nodes and branches, appear to strongly co-vary within species in the figures. I suspect they are developmentally strongly correlated and thus just two sides of the same coin - if this is the case, they should be combined for analysis or the Mantel tests corrected for multiple comparisons.

Other comments: The analysis is focused on comparing two closely related subspecies that, I believe, differ mostly in their floral traits. Have there been any previous studies comparing vegetative traits between the subspecies in wild 


\section{Biol}

populations? If not is this a new contribution to this system? If yes, it would be very nice to include in the Methods to provide some background, and to explain how traits vary in the common garden when growing at the same altitude compared to in the wild.

Along those lines, the main traits that differ between the two taxa are floral traits, yet the current study is focused on vegetative traits. This is fine and interesting but it requires some justification - why exclude reproductive traits if their comparison could also be interesting in this particular system?

Given that the paper is focused on the differential adaptation patterns between the two subspecies, it could be good to add a bit more about what is known (or unknown) on what keeps the two subspecies separate. I see that there is some explanation on this in the response to the previous round of reviews, so it should be relatively straightforward to include a justification for it here. It seems important to rule out that similar mechanisms could be affecting vegetative traits as well.

Line 161-162: unclear, please be more explicit

Minor comments:

Please carefully check the manuscript for style and English. Here are just a few examples: - repeated use of the Word "potential" in the Abstract - word choice: "combined" in line 48; "participates" in line 119; "all traits" in line 340; "comforted" in line 369 Fig S1: please use the colour codes used in other figures to show the two subspecies Line 165: "across the entire spatial distribution" is unclear Lien 174: sentence on maternal effects: rephrase for clarity Line 192: some of this information on the traits is already in the Introduction so seems redundant; please explain instead how/when traits were measured. Line 221: is the "plastic container" the randomized block? Line 272: Mantel tests - specify that they were run separately for each subspecies Table 1 legend refers to analysis in JMP? Paragraph starting in line 339 needs revision; there is some repletion and awkward phrasing Please check figure numbers across manuscript

\section{Author's reply:}




\section{Biol}

Dear recommender, all files (track changes revised version, clean pci evol biol pdf template, data and R analysis code, LaTeX code) can be found on zenodo open access file deposit 10.5281/zenodo.3821949

Thank you for the opportunity to submit our revised manuscript.

We took into account all your comments and the comments of the two reviewers (in blue in the following text). We brought a particular attention to clarifying the methods (QST calculation).

The suggested modifications improved the quality of the paper. We thank you and the reviewers for your comments and hope that you will find the manuscript suitable for recommendation. Please find below a detailed response to technical requests, recommender comments, and reviewers comments.

We uploaded a version of the revised manuscript where modifications appear in the track changes format so that they can be visualized easily. We also uploaded a clean Latex version of the revised manuscript using the PCI Evol Biol template.

Looking forward to hearing from you.

Regards, for the authors, Benoit Pujol

\section{RECOMMENDER'S COMMENTS}

Abstract: the words " potential » or " potentially » appear 6 times in the abstract. It is good to be cautious but surely some of these could be reworded (check lines 21-24 out in particular). Done, we have deleted and/or replaced 4 of them.

Line 249: I am not sure what "linearized" means here. Done, changed to "normalized".

Line 263: replace 'last' with 'latter'. Done

Lines 282-285: this latter analysis seems to be the most comprehensive, and the one you really need to address your main questions. What is the point of the other Mantel tests described above? We agree that the last Mantel test that we 
performed (population pairwise QST and population pairwise altitude differences while controlling for neutral genetic differentiation) is the most important to address our main question. However, Mantel tests are only correlation tests. We believe that the step by step procedure that we used here strengthen our results. The correlation between Qst and Fst was not significant and the correlation between Qst and altitude was significant. As a result, the results of the last Mantel test appear more reliable.

Lines 312-317: Could you confirm that the difference in the correlation between trait values and altitude between the two taxa is not due differences in the variance in altitude across populations for each taxon? Is variance in altitude the same for both taxa? The variance in altitude between subspecies was quite consistent. Yes, we can confirm that the variance in altitude was not significantly different between subspecies (anova p.value= 0.4 ). We added text to clarify this point in the methods (LL 163-165 and supplementary information).

Lines 332-336: do you mean that the results obtained via the examination of overlapping confidence intervals are fully consistent with those obtained by the bootstrapping method? If so, this is not entirely clear from this sentence. Yes, this is what we meant. We have modified the sentence to clarify this point LL348-352.

Lines 384 and 385: replace two instances of "less" with "fewer". Done

TECHNICAL COMMENTS Additional Comments of the managing board: Mandatory modifications. As indicated in the 'How does it work?' section and in the code of conduct, please make sure that: -Data are available to readers, either in the text or through an open data repository such as Zenodo (free), Dryad or some other institutional repository. Data must be reusable, thus metadata or accompanying text must carefully describe the data. Done (data files deposited on ZENODO) 10.5281/zenodo.3821949

-Details on quantitative analyses (e.g., data treatment and statistical scripts in R, bioinformatic pipeline scripts, etc.) and details concerning simulations (scripts, codes) are available to readers in the text, as appendices, or through an open 
data repository, such as Zenodo, Dryad or some other institutional repository. The scripts or codes must be carefully described so that they can be reused. Done ("read me" listing the data and analysis files and ready-to-run-analyses ( $R$ protocols) calling for the data files automatically are deposited on ZENODO) 10.5281/zenodo.3821949

-Details on experimental procedures are available to readers in the text or as appendices. Done

-Authors have no financial conflict of interest relating to the article. The article must contain a "Conflict of interest disclosure" paragraph before the reference section containing this sentence: "The authors of this preprint declare that they have no financial conflict of interest with the content of this article." If appropriate, this disclosure may be completed by a sentence indicating that some of the authors are $\mathrm{PCI}$ recommenders: "XXX is one of the $\mathrm{PCI} X X X$ recommenders." Done

In order to reach a better referencing and greater visibility of your recommended preprint, we suggest you to do the following modifications: - add the following sentence in the acknowledgements: "Version 3 of this preprint has been peerreviewed and recommended by Peer Community In Evolutionary Biology (https://doi.org/10.24072/pci.evolbiol.100097)" Done

- in the Zenodo metadata "Notes" you can indicate: "This article has been peerreviewed and recommended by Peer Community In Evolutionary Biology (https://doi.org/10.24072/pci.evolbiol.100097)" Done

- in the Zenodo metadata "Related article" you can indicate: "Reviewed by PCl Evol Biol 10.24072/pci.evolbiol.100097" Done

Optional modifications. ==> We advise you to use templates (word docx template and a latex template) to format your preprint in a $\mathrm{PCl}$ style. This is optional. Here is the links of the templates: https://peercommunityin.org/templates/ Done

For word template, please be careful to correctly update all text in these templates (doi, authors' names, address, title, date, recommender first name and family name ...). Please be careful to choose the badges "Open Code" and "Open 
Data" only if appropriate (in addition to the "Open Access" and "Open PeerReview" badges). If some of the reviewers are anonymous, indicate for example "Albert Ayler and two anonymous reviewer". Indicate in the "cite as" box the right version of your preprint. It is version 3.

For Latex template, main.tex and sample.bib should be filled. Please be careful to choose the badges "Open Code" and "Open Data" only if appropriate (in addition to the "Open Access" and "Open Peer-Review" badges). Preambule_xxx.tex should be modified (comment lines 115, 117) to select badges. If some of the reviewers are anonymous, indicate for example "Albert Ayler and two anonymous reviewer". In sample.bib, indicate the right version of your preprint. It is version 3. Done

\section{REVIEWS}

REVIEWED BY SANTIAGO C. GONZALEZ-MARTINEZ, 2020-04-10 10:08 In this second round of revision, I only have a major comment. I am afraid that the equation provided to compute Qst, $\mathrm{Vb} /(\mathrm{Vb}+2 \mathrm{~h} 2 \mathrm{Vw})$, is wrong. The correct denominator of this equation is the sum of the variance among populations ( $\mathrm{Vb}$ ) and twice the additive variance within-populations, which in your case (full sibs) is twice the family variance $(\mathrm{Vw})$, thus the equation should read: $\mathrm{Vb} /(\mathrm{Vb}+2 \mathrm{Va})$ or $\mathrm{Vb} /(\mathrm{Vb}+4 \mathrm{Vw})$ or $\mathrm{Vb} /(\mathrm{Vb}+2 \mathrm{~h} 2 \mathrm{Vp})$, with $\mathrm{Vp}=\mathrm{Vw}+\mathrm{Vres}$. I tried to check out if this is just an erratum by recomputing Qst from the variance components from Table S3 but Qst values do not seem to fit with those in Figure 4 (Qst values themselves are not reported in the manuscript) in any way I compute them. Please, check this out, as it may imply major revisions to the manuscript. You are right; there was a transcription error in the methods. Qst should have appeared as QST = Vb / ( Vb $\left.+2 h^{2}(\mathrm{Vw}+\mathrm{Vres})\right)$, as you noted. Very worried, we screened all our $\mathrm{R}$ code and detected an error (the Vres was there but the factor 2 had jumped). We re-ran all the analyses. It did not change the results much, the values barely changed. The only noticeable change was one QST value in A. m. pseudomajus, which P-value was not significant by much, that became nonsignificant. Nothing changed for A. m. striatum. In fact, this makes our conclusion 
on the potential absence of local adaptation in A. m. pseudomajus more consistent, which indirectly strengthened the observed contrast with the potential presence of local adaptation linked to altitude in A. m. striatum. This reinforced our conclusion that different mechanisms are at work in the two subspecies. For the sake of clarity and transparency, we added the values of the QSTs in Table S3. We also provided the code and data with the MS.

Minor comments: Ln 35. Replace "subspecies" by "subspecies divergence". Keywords: done

Ln 49-51. Can you be a bit more specific? Done, we added text to provide ecological context to the citations made in the text (LL 48-58)

Ln 100-102. Not clear to me why you would not expect shorter internode length at high altitudes, as I would expect than to only height but also growth rate would be lower (but I am not an expert on this, so I am probably wrong). In any case I like very much this paragraph setting the expected trait variation with altitude. In fact, a lower biomass might not be due to a lower growth rate at a higher altitude. We agree that on the one hand, this may be expected because the final biomass or height should be lower at a higher altitude. However on the other hand, plants are also expected to germinate later at a higher altitude, and consequently to have a shorter growth period. These plants might then have a higher growth rate at a higher altitude to reach a lower vegetative biomass required to reproduce in due time.

Ln 111. 'based on trait heritability', a bit weird to express it in this way... perhaps better to say 'based on partition of genetic variance'? Done

Ln 138. I guess that you estimated genetic differentiation using Fst? Can you add this between parentheses? Done

Ln 159. It is perhaps not very important for this study, as climatic data is only used to characterise the populations, but worldclim does notably bad in mountain regions (in particular for precipitation) and alternative climate databases are preferable. We agree that wordclim might lack some precision in mountain regions. It was nevertheless used satisfactorily in previous work to characterize 
the ecological niche of $A$. majus at the level of populations, across the altitudinal gradient in the Pyrenees, and across the species geographic distribution (Khimoun et al. 2013. Evolutionary Ecology 27: 51-64.). We'd rather keep this approach that has been proof tested in previously published work.

Ln 205. I assume that you mean '...the 637 plants that germinated'? (As there should have been $40 \times 24=960$ pots). Please, specify. Yes indeed, we clarified this point by adding "that germinated" in the text.

Ln 221/230/241. '... plastic container ('block') as a random...' Done

Ln 231. '...where Vw is the family...' Done

Ln 243. Please, see my major comment above. Hope it is just an erratum! See response to major comment above.

I think you would need to report Qst values somewhere in the Results (perhaps adding these values to Table S3?). Done, Qst values have been reported in Table S3.

I also miss some discussion on these values and the comparison with values for other plants in the literature. In the results section, we added text to discuss overall Qst -Fst differences found in our study and those found in the literature (L359-366). We are not aware of any other studies using a Qst-Fst approach on this species or any other Antirrhinum species.

Ln 323. 'Several traits had similar... Done

Ln 366-367. I would say that signatures of potential selection were found for only few traits. Do you think this is because low power of the study (Cls for Qst are large, judging by Figure 4) or does it truly reflect the species' biology? We now mention that "signatures of potential selection were found for only few traits" L392 in the discussion. We added text in the results LL359-366) where we acknowledge the low power of our approach. Significant QST-FST differences were likely very conservative in our study. One must note that our conclusion about A. m. striatum and A. m. pseudomajus differences is robust to this lack of power. 
Ln 389. Should this section not come before the previous one? (As it talks about overall differences in adaptation patterns across subspecies) We'd rather keep it that way. We adopted this logical structure following the suggestions made during the first round of reviews. We believe that this logical structure of the narrative is well suited as it goes from observed global patterns (altitude signal) to observed detailed patterns (subspecies and populations) to mechanisms underlying detailed patterns (ecological and then population genetic aspects).

Overall, the Discussion section is still a bit reiterative and, towards the end, it puts too much emphasis on adaptive divergence. We chased the reiterations and removed them as much as we could as to lower the emphasis on adaptive divergence.

This is surprising as adaptive divergence was found for only a handful of traits in this study. I think that a section devoted to why the study did not identify adaptive divergence in more traits (some of them with obvious adaptive value, e.g. SLA) and why altitude did not seem to be a major factor for adaptive divergence in this system would be desirable. We agree that these results were surprising. We added text to discuss (i) the power of the analysis (see comment before), (ii) whether other studies found similar QST-FST differences (see comment before), (iii) had already discussed expected trait adaptations in response to altitude in the introduction in the previous version (including SLA), and (iv) added text in the discussion to address the lack of altitudinal signal for SLA in both subspecies (LL413-415). We'd rather not speculate on the adaptive value of A. majus traits that our statistical analysis did not identify as potential candidate traits involved with adaptation. We'd also rather not speculate on the reasons "why altitude did not seem to be a major factor for adaptive divergence" because we already discuss our results that altitude is involved to some extent with adaptive divergence. We hope that additional studies will be able to bring further elements to answer these questions.

Figure 2's legend. No need to report the regression fits as they are not significant. Done 


\section{Biol}

Figure 5 needs updating (these linear correlations were removed from the manuscript, thus should not be reported in this figure). Also, why Fst in the y-axis? Done, we removed the regression equations, and added text in the legend to specify that these graphs illustrate the relationship formally tested by mantel tests and that the Fst on the $y$-axis refers to the grey dots.

All in all, this is a very nice paper and hope that authors will be able to deal with the few revisions that are still needed (and in particular to check out that Qst values are indeed properly computed).

REVIEWED BY ANONYMOUS REVIEWER, 2020-04-09 13:31 The manuscript by Marin et al. has changed greatly after the revision, so that many of the previous comments by reviewers do not apply anymore. In my opinion the new version is a clear improvement, based on what I read in the previous reviews. My review below is thus independent from those.

The paper is an interesting contribution to our understanding of plant adaptive variation (or lack thereof) along elevation gradients and of how recently-diverged taxa could be responding differently to similar environmental pressures. The authors use an Fst/Gst approach, based on a common garden and previous field studies, to study patterns of local adaptation of two subspecies of snapdragons. The approach is sensible in cases like this where reciprocal transplants are difficult. The paper is overall clearly written but there are some aspects of the analysis and results that need clarification:

- Values of narrow sense heritabilities are estimated in the greenhouse across all populations within each subspecies, rather than for each population. Please justify why this done, given that all subsequent analyses are based on population comparisons, and that we know that $\mathrm{h} 2$ values are population-dependent. The authors mention in line 234 that caution must be taken with $\mathrm{h} 2$ values, but it is not clear how they are being cautious in the analyses that follow. We modified the text LL248-252 to clarify this point. In brief, full sib designs provide less precise heritability estimates than full pedigree based estimates. To increase 
precision, we traded off the detailed information at the level of populations and produced only one global estimate. We then took into account the uncertainty around $\mathrm{h}^{2}$ by providing its $95 \% \mathrm{Cl}$ (calculated by using a parametric bootstrap method). We were cautious not to interpret trait $h^{2}$ values in terms of quantified evolutionary potential to respond to selection or to compare them between traits in the discussion.

- The text explains that pairwise relationships were tested with Mantel and partial Mantel tests, but legends of Fig 2 and Fig 5 include regression equations and $p$ values - please clarify what the analysis was. We removed the equations produced by linear regression from Figures 2 and 5 . These analyses were removed from the manuscript after the previous round of reviews. Figure legends should have been updated accordingly. Plot figures were kept to illustrate graphically informal trends in the data that were formally tested by Mantel tests.

- I would expect that some of the traits included in the analysis are correlated to each other, notably the number of nodes, branches and internode length. The two traits that are found to vary with altitude, nodes and branches, appear to strongly co-vary within species in the figures. I suspect they are developmentally strongly correlated and thus just two sides of the same coin - if this is the case, they should be combined for analysis or the Mantel tests corrected for multiple comparisons. We added text LL410-412 to acknowledge that these two traits are strongly correlated because axillary buds producing branches are located at nodes. We believe that using a correction for multiple comparisons (eg. sequential Bonferroni correction) is not useful here because only one trait (number of nodes) was considered significant. We flagged another trait on the list as being marginally significant. No other trait was found significant. Using a correction for multiple comparisons here such as the sequential Bonferroni correction would therefore give the exact same result.

Other comments: The analysis is focused on comparing two closely related subspecies that, I believe, differ mostly in their floral traits. Have there been any previous studies comparing vegetative traits between the subspecies in wild populations? If not is this a new contribution to this system? If yes, it would be very nice to include in the Methods to provide some background, and to explain 


\section{Biol}

how traits vary in the common garden when growing at the same altitude compared to in the wild. This is indeed to our knowledge a new contribution to this system. We are not aware of studies having measured vegetative traits of $A$. majus natural populations in the wild or in experimental gardens. We therefore cannot compare. As you might already know, research on A. majus conducted to date was mainly focused on floral traits.

Along those lines, the main traits that differ between the two taxa are floral traits, yet the current study is focused on vegetative traits. This is fine and interesting but it requires some justification - why exclude reproductive traits if their comparison could also be interesting in this particular system? We added text in the methods to clarify this point LL212-215. Following the reviewers' rightful request on the first round of peer reviews, we have split the global data set in two, one for each subspecies. As a consequence, we had not enough statistical power to analyze reproductive traits because less data was available (not all plants that grow flower).

Given that the paper is focused on the differential adaptation patterns between the two subspecies, it could be good to add a bit more about what is known (or unknown) on what keeps the two subspecies separate. I see that there is some explanation on this in the response to the previous round of reviews, so it should be relatively straightforward to include a justification for it here. It seems important to rule out that similar mechanisms could be affecting vegetative traits as well. We added text LL151-153 to clarify that frequency dependent selection based on flower colour maintains A. majus subspecies separate. It would however be speculative "to rule out that similar mechanisms could be affecting vegetative traits" in the absence of a dedicated study and in the presence of evidence [from our study] for vegetative differentiation between subspecies [that is by definition correlated to flower colour].

Line 161-162: unclear, please be more explicit We added text L172 to clarify that as a result of sampling populations on different valley and summits, "populations with similar elevation are not geographically closer." 


\section{Biol}

Minor comments: Please carefully check the manuscript for style and English. Done

Here are just a few examples: - repeated use of the Word "potential" in the Abstract This was modified

word choice: - "combined" in line 48; changed to "combined the information from"

- "participates" in line 119; changed to "contributes to"

- "all traits" in line 340 removed

- "comforted" in line 369 changed by "support"

- Fig S1: please use the colour codes used in other figures to show the two subspecies Done

- Line 165: "across the entire spatial distribution" is unclear removed

- Lien 174: sentence on maternal effects: rephrase for clarity . Done, rephrased to: "This intermediate generation of plants grown in controlled conditions allowed us to reduce potential maternal environmental effects that could have otherwise affected plants grown from seeds sampled in the wild." LL184-185

- Line 192: some of this information on the traits is already in the Introduction so seems redundant; please explain instead how/when traits were measured. Done, we now presented how/when traits were measured.

- Line 221: is the "plastic container" the randomized block? Yes indeed, we added "block" into brackets.

- Line 272: Mantel tests - specify that they were run separately for each subspecies. Done

- Table 1 legend refers to analysis in JMP? Every analysis was done in R on this new version of the manuscript, the legend needed to be updated. We corrected this. 


\section{Biol}

- Paragraph starting in line 339 needs revision; there is some repletion and awkward phrasing Done, rephrased to "Mantel tests showed no relationship between population pairwise QST and FST for most of traits (Table 2). Only population pairwise QST for the germination date in A. $\mathrm{m}$. striatum was significantly correlated with population pairwise FST." LL368370

- Please check figure numbers across manuscript Done

Download author's reply (PDF file)

\section{Revision round \#1}

2018-10-16

Dear Sara Marin,

I would first like to apologize for the time needed to return this decision, which is partly attributable to the difficulty of finding available reviewers in the middle of summer. Your preprint has now been read carefully by two experts (including Sophie Karrenberg (SK), who signed her review) and by me. We all agree that your manuscript addresses and important evolutionary question (patterns of adaptation to altitude), and that the experimental design was appropriate to address this question, such that I would ultimately be willing to recommend this preprint provided you can address the issues raised by the reviewers. Here are the most critical issues I identified, some of which were raised independently by both reviewers:

(1) Definition of local adaptation: both reviewers and I were lost with, or at the minimum confused by, your distinction between local adaptation (the meaning of which seems to vary throughout the paper, see comments by SK) and adaptation to latitudinal gradients, which involves local adaptation in the broad sense, such that individuals with the highest fitness in one location are on average those originating from this location. It is really important that this point is clarified. This may include for example specifying whether you consider local adaptation as a 
pattern or as a process or both. Another option for clarification would be to follow the first suggestion of SK, which is to focus more on biology and the question of adaptation to altitude, and how to disentangle it from adaptation to other environmental variables, which may vary in space at a finer grain than altitude (or not, see comment 7 by reviewer \#2)

(2) SK is worried that pooling the two subspecies might bias your analyses. There again, it is central to provide a solid justification for this pooling

(3) Methodological / statistical issues: both reviewers had several additional questions and suggestions regarding the statistical analyses, which I will not fully list here (although they all deserve attention), but which include for example: (i) How could you estimate the within population additive genetic variance, when the number of individuals and the number of families seem to be similar (i.e. about one individual per family)? (ii) How was the significance of the relationship between Fst (or Qst) and altitudinal differences tested in Figure 3? (iii) On the same figure, can the statistical significance of the differences in slopes be tested? More generally, the two reviewers often requested more details on the methodology (e.g. Qst-Fst comparisons, quantitative genetics analyses...), and I agree with them that some key information is sometimes missing.

(4) While the "Methods" section could be expanded, the two reviewers and I agreed that the text could also be shortened at places, particularly in the discussion.

Thank you very much for submitting your preprint for recommendation in $\mathrm{PCl}$ Evolutionary Biology. I hope you will find this feedback, and particularly the very thorough reviews of the experts, useful to revise your manuscript.

Best regards Emmanuelle

Preprint DOI: https://doi.org/10.1101/385377

Reviewed by Santiago C. Gonzalez-Martinez, 2018-08-07 20:44

Download the review (PDF file)

Reviewed by Sophie Karrenberg, 2018-08-28 18:08 
Your manuscript on altitudinal divergence in snapdragon plants treats an interesting and timely question mostly with appropriate methods. However, I do have several difficulties to evaluate the quality of this research because important information appears to be missing from the manuscript. This concerns: I), most importantly, whether there were genetic or phenotypic differences between two subspecies that were apparently pooled here, according to Material and Methods and II) the experimental design such as spatial arrangement in the garden (how plants were arranged in how many containers) that should be taken account of as a random effect in the analyses, as well as crossing design and number of individuals per family used (see comments below). Regarding the subspecies, an explicit analysis would be most helpful (phenotypic and genetic differentiation, for example PCoA or structure analysis, potentially presented in the supplementary material). Pooling of the subspecies would probably only be appropriate if there are no considerable phenotypic differences or genetic differentiation between them. Fst in these analyses is used to reflect putatively neutral genetic differentiation -- this might be difficult assumption if the dataset includes subspecies that are partially reproductively isolated from each other. The description of the subspecies in the method section as well as the citations do fuel this concern. Differences in habitat and flower color between subspecies are described -even pollinator-mediated reproductive isolation could be present in this system. Very careful and convincing analyses and explanations would be needed to motivate the pooling. And -if there are no differences between subspecies, would that not suggest that there might just be a flower color polymorphism? It is important to note that, without this potentially troublesome pooling of subspecies, the number of populations would probably be too low for the present analysis and this is why this issue is so critical. Provided that pooling can be justified, and the design clarified, this manuscript could become a good one, however, further improvements in the analysis and presentation are also needed. The main changes that I believe are necessary are:

1) Focus on biology. The paper would be more interesting if focus was shifted from methods and general interpretations to the biology of altitudinal adaptation. This would be facilitated by presenting the trait-altitude correlations first. These 
results are interesting for all traits, currently they are only presented for 3 out of 13 traits. From that you could move on to more complex analyses to identify the underlying causes of such trait clines. In the current version, there is overall rather little on HOW the traits change along altitude, for example, there are no details on leaf traits, even though there is a lot of literature of variation in SLA (see citations in literature mentioned below). It would also be very good if explicit expectations for all traits or for trait groups, based on the literature, could be added in the introduction. I would also appreciate an introduction ending in specific questions.

2) In the methods part, you write that you used the between family variance component to estimate the within population additive genetic variance for a trait, as is commonly done. However, Table S1 states, for reproductive traits, the total number of families is 372 and the number of individuals is 380 , such that you cannot have had more than one individual per family in most cases, unless you used a subset of families with more individuals (I could not find information on the number of individuals per family). With 1 individual per family it should be very difficult or impossible to estimate the between family variance component. In Table S3 you do not give the within family variance component so I wonder whether what you state as the within population variance component really is the between family variance component. This issue is in need of clarification. It would also be good if you could explain why you have fewer measurements for reproductive traits than for vegetative traits.

3) Methods, QST - FST. I found the description of the QST - FST comparisons, using expected distributions, unclear and in part contradictory (see comments in the text). In particular, it is not clear to me how you arrived at the confidence intervals for QST on Figure 2. It would fit better if you presented the distribution of expected values for QST together with the observed values, this is the method you stated in the methods section. Alternatively, you could change the methods section.

4) Analysis of pairwise data: the Methods section describes the commonly used and suitable analyses for correlation tests among matrices (pairwise data), Mantel tests and partial Mantel tests, controlling for the effect of neutral genetic 
divergence. In the results however, a regression analysis is presented (Fig. 3) that is not suitable for this type of non-independent data points. The text states that the Mantel test was used to assess significance of the regression - this is not possible. The actual data points are also not displayed, this is a bit worrisome due to the subspecies issue. My suggestion is to re-work this analysis according to the methods described and to display the data points on Fig. 3.

5) (Local) adaptation, interpretation. The interpretation of what constitutes local adaption is highly variable throughout the manuscript. At some places, the common cautious interpretation of QST - FST analyses, that they can provide hypotheses on traits that may have adaptively diverged due to selection (see for example review by Leinonen et al. 2013, Nature Reviews Genetics) is used. At quite some other places, however (abstract, introduction, discussion, marked in detailed comments), it appears that local adaptation is inferred whenever trait divergence cannot be attributed to either the altitudinal gradient or neutral divergence. I cannot be sure that the text is really meant that way, of course, but I testify here that it reads that way. This interpretation would be incorrect (genetic drift and many other processes could be responsible for trait divergence instead). Any text that can potentially be misunderstood is probably better changed.

6) Interpretation of the results on germination. The corresponding paragraph in the discussion unfortunately appears contradictory and the two types of results, overall QST - FST and pairwise QST - FST have not been compared (see comments in the text). Moreover, the text also appears as if it was not clear to the authors that a lack of quantitative genetic differentiation in traits does not exclude phenotypic plasticity (i.e., this is not really an alternative interpretation). It seems that this part needs adjustment.

7) Interpretation of genetic divergence. You invoke reproductive isolation, when it appears that isolation by distance would be a sufficient interpretation, unless you refer to the subspecies differences here (see comments above and in the text). However, this cannot be evaluated from the data currently presented, as details on genetic differentiation are missing (see above). I suggest that this interpretation is re-worked one a more detailed analysis is presented. 
8) Literature. The manuscript would much benefit by adding more literature on the analysis of altitudinal gradients and transplant experiments, for example: Luo, Y., A. Widmer, and S. Karrenberg. 2015. The roles of genetic drift and natural selection in quantitative trait divergence along an altitudinal gradient in Arabidopsis thaliana. Heredity 114:220. Halbritter, A. H., S. Fior, I. Keller, R. Billeter, P. Edwards, R. Holderegger, S. Karrenberg, A. R. Pluess, A. Widmer, and J. M. Alexander. 2018. Trait differentiation and adaptation of plants along elevation gradients. J. Evol. Biol. [Disclaimer: these are papers I am an author on-- but they really are related here... as are many of the citations in them].

9) Methods are not really new. Throughout the intro and the discussion, the paper reads as if this mainly is a method advancement. This appears somewhat exaggerated, as similar methods have been used before and since a longer time ( $>5 \mathrm{yrs}$ ). I feel that the work of others should be acknowledged much more here, only few citations on such studies that are able to identify selective agents are given (for example, Hangartner et al).

10) Shortening. I find that the text could likely benefit from shortening, in particular in the introduction and discussion, probably to at least to $2 / 3$ of its present length. I have indicated several parts that appear repetitive in the detailed comments.

11) More comments. I have made comments similar to the above comments as well as many further detailed comment directly on the text using pdf commenting tools in Adobe Acrobat. I am not repeating these detailed comments here. Such a list of details would be very difficult to write and read, because the text did not include any line numbers to refer to.

I hope that you will find my comments clear and helpful for further revisions of your work.

With best wishes, Sophie Karrenberg

Download the review (PDF file)

\section{Author's reply:}


कि $\mathrm{PCl}$ 